\title{
Diabetic kidney disease and its association with macrovascular disease in diabetic patients
}

\author{
Elena García Fernández, Gonzalo Allo Miguel, Lucas Arroyave Villa, \\ Guillermo Martínez Díaz-Guerra, Federico Hawkins \\ Endocrinology Service, 12 de Octubre University Hospital.
}

\section{Introduction}

It is well known that diabetes, especially type 2 (T2DM), confers a substantial burden of macrovascular disease. Previous studies suggest a link between retinopathy and macrovascular events in diabetes. However, just a few studies have investigated the association between peripheral diabetic kidney disease (DKD) and cardiovascular disease.

\section{Aim}

The purpose of this study is to investigate whether the presence of peripheral neuropathy is associated with macrovascular disease in diabetes patients.

\section{Patients and methods}

98 diabetic patients (48 male/50 female) were included in the study. DKD was confirmed by the presence of moderately or severely increased albuminuria. Adverse cardiovascular events were collected, including: cardiac events, left ventricular hypertrophy (LVH), peripheral vascular disease (PVD) and stroke. Statistical analysis: Chisquare test was used to compare the existence of macrovascular complications between groups with and without DKD.

\section{Results}

68 patients with type 2 diabetes and 20 with type 1, with mean age $(52.58 \pm 20.70)$ and mean $\mathrm{HbA} 1 \mathrm{c}(11.21 \pm 2.33 \%)$, were included in the study. DKD was diagnosed in 25 patients: 17 with moderately increased albuminuria and 8 with severely increased. DKD was significantly correlated with the existence of, al least, one macrovascular complication $(p<0.02)$. Significant differences between the groups with or without DKD were found in the rate of: cardiac events $(32.00 \%$ and $9.84 \%$ respectively; $p=0.01)$; LVH $(36.00 \%$ and $11.11 \%$ respectively; $p<0,001)$ and PVD $(20.00 \%$ and $13.33 \%$ respectively: $p=0.04)$. These differences remained significant after adjusting for age, sex, hypertension, smoking habit, $\mathrm{HbA} 1 \mathrm{c}$, duration of diabetes and body mass index. No significant differences in the rate of strokes.

\begin{tabular}{|c|c|c|c|}
\hline & $D K D-(n=65)$ & $D K D+(n=25)$ & $p$-value \\
\hline PVD & $9(13.33 \%)$ & $5(20.00 \%)$ & 0.04 \\
\hline Cardiac Events & $4(9.84 \%)$ & $8(32.00 \%)$ & 0.01 \\
\hline LVH & 7 (11.11\%) & 9 (36.00\%) & $<0.001$ \\
\hline
\end{tabular}

Table 1. Patients with diabetic kidney disease have significant higher rates of peripheral vascular disease, cardiac events and left ventricular hypertrophy.

\section{Conclusions}

Our results show significant correlation between DKD and the existence of one or more macrovascular complications. Moreover, we have found that diabetic patients with DKD present significant higher rates of cardiac events, LVH and PVD than diabetic patients without DKD. Despite the limitations of the study, our results suggest that early detection of DKD might be a valuable component of macrovascular risk assessment in diabetic patients. 